\title{
Placenta percreta a case study and literature review
}

\begin{abstract}
The placental acretism, defined as the abnormal insertion of chorionic villi in the myometrium, is one of the main causes of maternal mortality. The key of the progress in the maternal-foetal results is the prenatal diagnostic, which is based fundamentally on the ultrasound scan, to perform a multidisciplinary programming of the treatment, conservative or surgical. We present a case study of a pregnant woman in which conservative treatment was performed initially with embolization of hypogastric arteries, leaving the placenta in situ after delivery, but that 5 weeks later the patient needed hysterectomy for sepsis due to necrosis tissue placenta.
\end{abstract}

Keywords: placenta accreta, conservative treatment, maternal morbidity, neonatal morbidity

\author{
Volume 4 Issue 5 - 2018 \\ Laura Revelles Paniza,' Susana Ruiz Durán,' \\ María del Mar Molina Hita,' Marta Revelles \\ Paniza, ${ }^{2}$ Gonzalo Ruiz Villaverde, ${ }^{2}$ Manuel \\ Barranco Armenteros, ' Alberto Puertas \\ Prieto' \\ 'Department of Obstetrics and Gynaecology, University \\ Hospital Virgen de las Nieves, Spain \\ ${ }^{2}$ Department of Diagnostic Imaging, University Hospital Virgen \\ de las Nieves, Spain
}

\begin{abstract}
Correspondence: Susana Ruiz Durán, Physician Specialist in Obstetrics and Gynaecology, Department of Obstetrics and Gynaecology, University Hospital "Virgen de las Nieves", Granada. Spain, Avd. Divina Pastora 9, Block 13, 4 B. 18012 Granada, Spain, Tel +34666462333.

Email sruizduran@hotmail.com
\end{abstract}

Received: June 20, 2018 | Published: September II, 2018

\section{Introduction}

Placenta accreta (PA) is the abnormal insertion of chorionic villi directly to the myometrium, with an absence of intervening deciduas. ${ }^{1}$ There are three types of PA: i) placenta accreta occurs when the placenta attaches directly to the myometrium with an absence of intervening decidua ( $78 \%$ of cases); ii) placenta increta is a deep invasion of chorionic villi into the myometrium ( $15 \%$ of cases); and iii) placenta percreta is a growth of the placenta completely through the myometrium, with perforation of the serosa (5\% of cases). The latter causes the highest maternal and perinatal morbidity and mortality. The incidence of PA is estimated in 1 over 2,500 deliveries and has increased in recent years. The antecedent of caesarean section and placenta previa are the main risk factors. ${ }^{2}$ Other factors involved include medical procedures on the uterus (e.g., previous myomectomy, uterine curettage and endometrial ablation), uterine malformations, and medical procedures, such as uterine embolization or pelvic irradiation. ${ }^{3}$ Because of its efficacy, reproducibility, lower cost and less time required for exploration, ultrasound is the primary method of evaluation and diagnosis of suspected PA. Magnetic resonance (MR) complements and supports the ultrasound diagnosis. ${ }^{4}$ The optimal management of this condition remains controversial. Although hysterectomy is an option for the retained placenta, leaving the placenta in situ with a conservative treatment such as uterine arteria embolism, mifepristone, methotrexate or hysteroscopy resection can also be performed when there is no haemorrhage and the patient wishes to preserve her fertility. ${ }^{5}$

\section{Case study}

In $31+5$ weeks, a 34-year-old woman with a history of eight gestations and four normal vaginal deliveries, one of them resulted in caesarean section, without any other medical interesting precedents, came to hospital with hydrorrea sensation, confirming her premature rupture of membranes (PROM). Gestation until that moment was normoevolutive with normal ultrasound scan of the first trimester and normal morphologic in week 20. During the evaluation ultrasound scan showed placenta accreta, with thin uterine musculature and disrupted by placental protrusions in the lower anterior uterine wall, placenta previa, loss of the retroplacental "clear space", confluent lagoons, giving a Swiss cheese like appearance, protrusion of the placenta into the bladder, increased vascularity of the uterine serosa and turbulent blood flow through the lacunae on Doppler ultrasonography.

Betamethasone was administered for foetal lung maturation and antibiotic prophylaxis for PROM. MR was requested (Figure 1) in order to better determine the extent and depth of the invasion, which confirms the presence of a placenta previa. MR showed that in the lower half of anterior wall uterine body, above the bladder, there was a bulging of the placenta on the myometrium, with interruption of the trilaminar morphology of the myometrium, suggestive of its infiltration, probably to the serosa. The upper wall of the bladder showed a discontinuous effacement, contacting extensively with the placenta, without identifying in this location the trilaminar morphology of the myometrium, which suggested infiltration of the same, without evident extension in the bladder lumen.

A body caesarean section (Figure 2) was chosen after 6 days, tubal ligation and uterus-ovarian ligament, leaving the placenta in situ with subsequent embolization of hypogastric arteries (Figure 3). During the intervention and in the immediate postoperative period, the patient did not need a blood transfusion. She had an acute respiratory failure that progressed favourably. After the stability of the patient and the decrease in BhCG values, the patient was discharged from hospital at 31 days with serial analytical controls twice a week. After 5 days, she returned to the hospital with fever $\left(38.5^{\circ}\right)$, abdominal pain, oliguria, leucocytosis with left deviation and elevation of acute phase reactants. Under a suspicion of placental necrosis, cultures of expulsion of placental remains were taken vaginally and antibiotic treatment started with Meropenem and Linezolid. A pelvic TAC was requested (Figure 4) that reported partial placental involution, with persistence of hypervascular areas and bladder extension, together with cysticnecrotic changes with gas compatible with abscess, so it was decided to perform a total abdominal hysterectomy (Figures 5) (Figure 6). In 
the postoperative period, administration of 6 packed red blood cells, 1 pool of platelets, fibrinogen, tranexamic acid and antibiotic treatment with Meropenem and Amikacin to cover E. coli and Proteus mirabilis isolated in the cultures carried out. The patient progressed favourably and was discharged 9 days after hysterectomy.

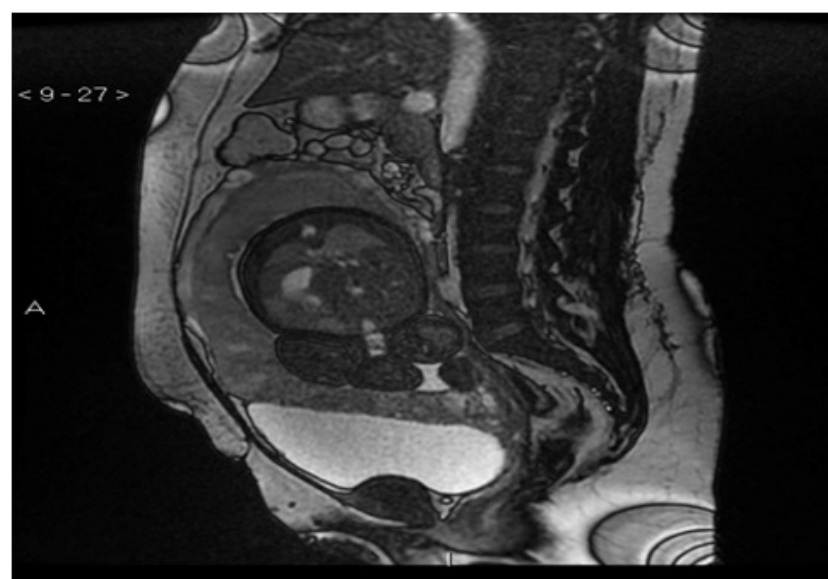

Figure I Sequence MR FIESTA T2 in plane sagital with placenta previa and percreta in the anterior wall of the uterine body and in the upper wall of the bladder.

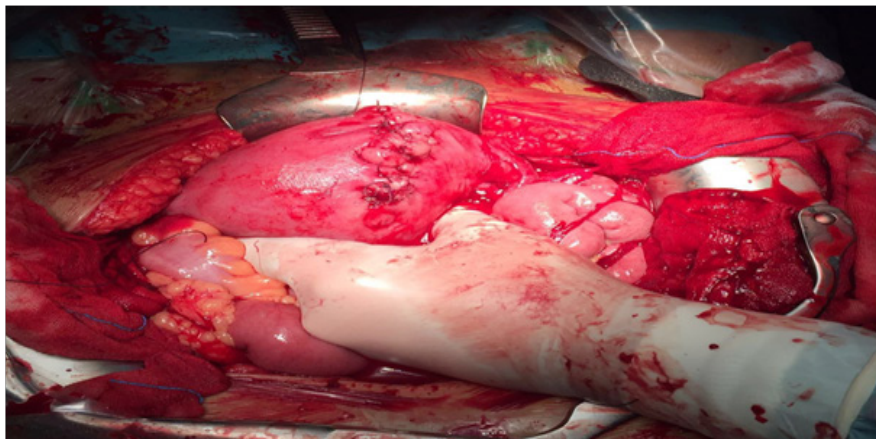

Figure 2 Cesarea corporal in gestation of 32 weeks per placenta percreta in a patient with a previous caesarean section.

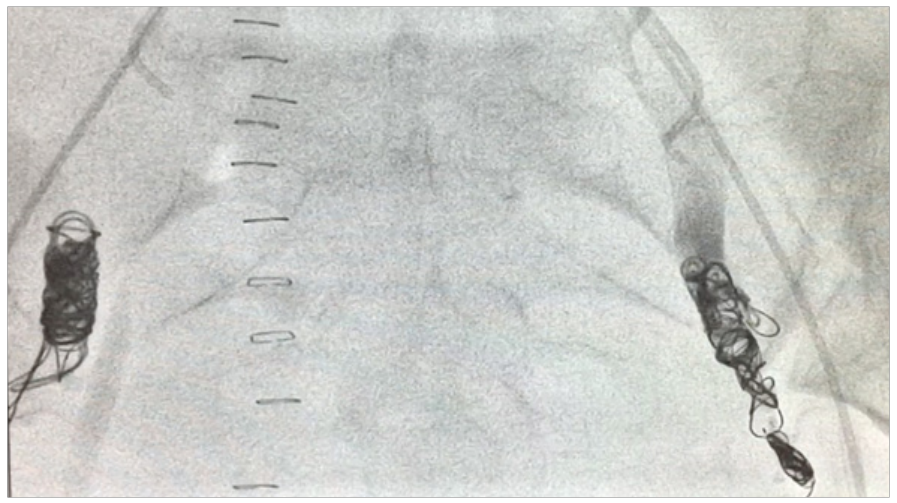

Figure 3 Hypogastric arteries embolism as conservative management placenta percreta.

\section{Discussion}

The origin of this placental anomaly would be generated by the partial or total absence of decidua basalis and the imperfect development in the fibrinous layer (Nitabuch's membrane), conditioning the development of accreta. The villi were fixed to the myometrium, invaded it or penetrated through it; one, some, or all cotyledons may be involved. The incidence of placenta previa and placenta accreta is increasing. Caesarean section is believed to constitute the main risk factor for the development of placenta accrete. The risk increases with the number of previous caesarean sections, even when they do not coexist with placenta previa, but it is in the association between both of them when the risk is more important ${ }^{2}$. In the largest cohort study of repeated caesarean deliveries (with 83,754 caesarean deliveries), published by Silver 2006, placenta accreta was in $0.24 \%$ at first caesarean, $0.31 \%$ at second, $0.57 \%$ at third, $2.13 \%$ at fourth, $2.33 \%$ at fifth, reaching values of $6.74 \%$ in the sixth caesarean section. In cases of placenta previa, the percentage of placenta accreta was $3.3 \%, 11 \%, 40 \%, 61 \%$, and $67 \%$ at the first, second, third, fourth, and fifth previous caesareans, respectively. ${ }^{2}$ Therefore, as in the present case, it is fundamental to highlight a diagnostic suspicion in the case that a placenta previa coexists with the antecedent of caesarean. This group should be subject to the utmost attention in order to discard AP. Fitzpatrick et al., ${ }^{6}$ published that $50 \%$ of the PAs attended in England between 2010 and 2011 were diagnosed intrapartum, with the increase in morbidity that this fact implies. This fact is even the more worrisome if we take into account that one third of these PA occurred in patients with a history of caesarean section and placenta previa in the current pregnancy.

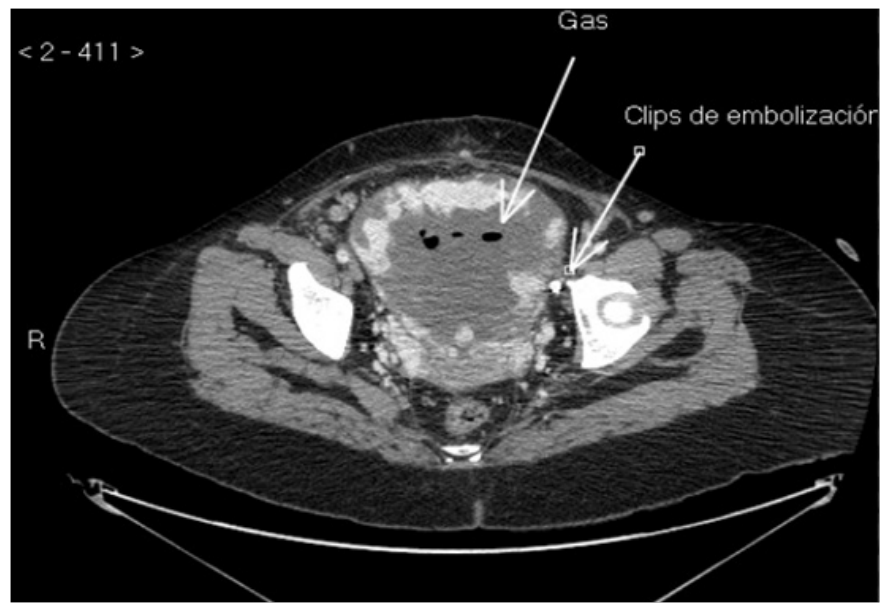

Figure 4 Pelvic TC after cesarean leaving placenta in situ. Partial involution of placenta is observed with cystic-necrotic changes with gas compatible with abscess.

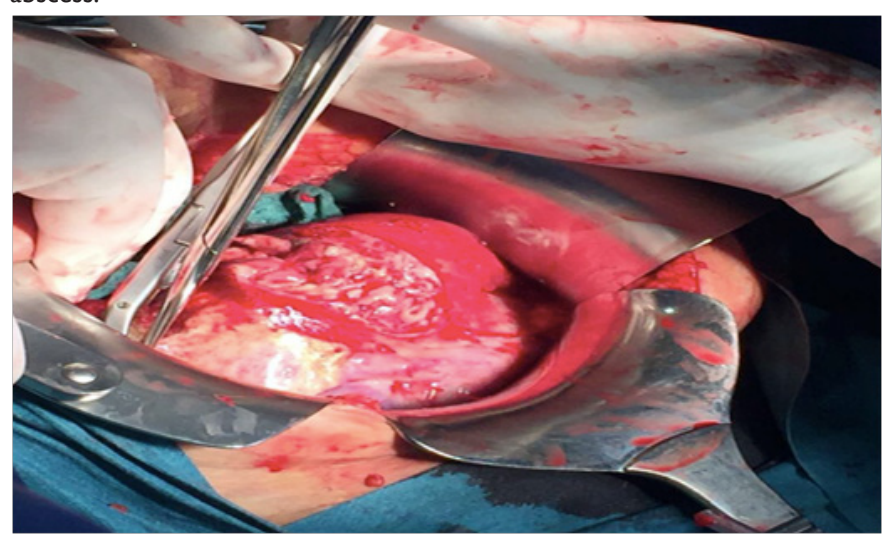

Figure 5 Hysterectomy in the necrosis tissue placenta context after embolism placenta percreta. 


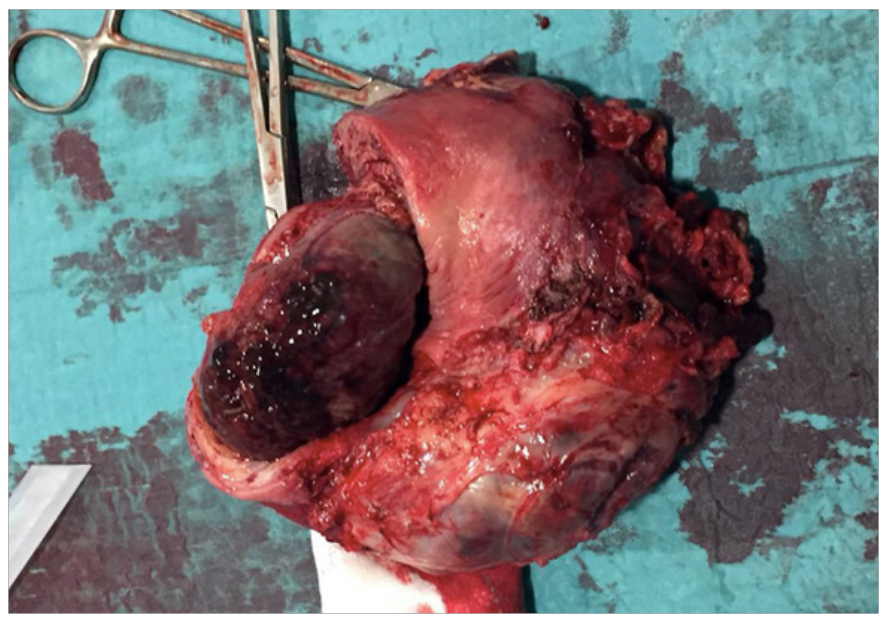

Figure 6 Necrosis tissue placenta after embolism placenta percreta.

Diagnosis before delivery is difficult. The overall performance for detection of placenta accreta was as follows: sensitivity and specificity $77-93 \%$ and $71-96 \%$ respectively. Grayscale ultrasonography is the first-line method to diagnose. D' Antonio et al., ${ }^{7}$ meta-analysis, that includes 32 studies with 3.707 pregnancies, shows that the overall performance of ultrasound for the antenatal detection of invasive placentation is as follows: sensitivity is $90.72 \%$ (95\% CI, 87.2-93.6); specificity is $96.94 \%(95 \% \mathrm{CI}, 96.3-97.5)$; $\mathrm{LR}+$ is $11.01(95 \%$ CI, 6.1-20.0); LR- is 0.16 (95\% CI, 0.11-0.23). The diagnosis is usually performed during the second and third terms, although cases with diagnosis in the first term have been described. This is the case presented here, in which the diagnosis was established in the third term of pregnancy. MR complements and supports ultrasound diagnosis, and it may be especially useful if the diagnosis is uncertain or inconclusive in high-risk placenta accreta pregnancies, placenta previa with location on the posterior or lateral wall, obesity, a possible invasion of the perimetrium or for planning the surgical procedure, in order to better determine the extent and the depth of the invasion. ${ }^{8}$ The importance of diagnosis of placenta accreta before delivery allows multidisciplinary planning in an attempt to minimize potential maternal or neonatal morbidity and mortality. In the present case the embolization was carried out by the Vascular and Interventional Radiology service after performing the caesarean section. In the second surgery, in which the total hysterectomy was carried out, the urologist participated along with the obstetric team. Likewise, both the radiological diagnosis and the stabilization and treatment of the patient by the anaesthesiology were key factors in the favourable evolution of the patient.

There are several treatment options in case of placental accreta: a) caesarean section hysterectomy with the placenta left in situ because removal of the placenta is associated with significant haemorrhagic morbidity; b) caesarean section with preservative treatment of the placenta (i.e., leaving the placenta in situ). Subsequent interventions include embolization of the uterine arteries followed by expectant management, and adjuvant medical treatment with methotrexate, $\mathrm{GnRH}$ analogues and prostaglandins; c) caesarean section with extraction of the placenta and conservative surgical treatment. If the goal is the uterus preservation and the extraction of the placenta, the techniques that we can carry out are the following: intrauterine balloon tamponade, B-Lynch suture, embolization or ligation of the uterine arteries, and/or the ligation of the anterior divisions of the internal iliac arteries, and partial excision of the invaded uterine wall. ${ }^{9,10}$ Both surgical and conservative treatments are associated with complications. Caesarean section and hysterectomy reports the following complications: $44 \%$ of transfusions of more than 4 packed red blood cells, $28 \%$ of coagulopathy, $49 \%$ admissions in intensive care, $5.7 \%$ of intestinal injury and $9.3 \%$ of reoperations. Conservative management is associated with a $15 \%$ transfusion of more than 4 packed red blood cells, $26 \%$ admissions in intensive care, $11 \%$ of hysterectomies, $4 \%$ of sepsis and $2 \%$ of uterine necrosis. ${ }^{11}$ In the present case, that reflects both management procedures, since initially we opted for a conservative treatment by embolizing the uterine arteries leaving the placenta in situ, initially achieving good control of the haemorrhage without the need for blood transfusion. Due to the subsequent complication with sepsis of uterine origin (placental necrosis), a total hysterectomy was performed, which required the transfusion of 6 packed red blood cells to stabilize the patient, which did not undergo any other complication, evolving favourably. Regardless the first chosen management approach, the best results (in terms of decreased maternal-foetal morbidity) seem to be associated with assistance by a multidisciplinary team. All this also requires a health centre with availability of resources and a blood bank in operation 24 hours a day, 365 days a year, and with capacity to treat patients with massive obstetric haemorrhage. All these aspects are found in high level centres, where it is mandatory to refer these patients. $^{12}$

\section{Conclusion}

Placenta accreta is associated with increased maternal morbidity, including blood transfusion, hysterectomy, admission intensive care unit, infection, and prolonged hospitalization. Neonatal morbidity is mainly associated with prematurity. Diagnosis of placenta accreta before delivery allows multidisciplinary planning in a specialised hospital in an attempt to minimize potential maternal or neonatal morbidity and mortality.

\section{Acknowledgements}

None.

\section{Conflict of interests}

Author declares there is no conflict of interest.

\section{References}

1. Adler E, Madankumar R, Rosner M, et al. Increased placental trophoblast inclusions in placenta accreta. Placenta. 2014;35(12):1075-1078.

2. Silver RM, Landon MB, Rouse DJ, et al. Maternal morbidity associated with multiple repeat cesarean deliveries. Obstet Gynecol. 2006;107(6):1226-1232.

3. Bowman Z, Eller AG, Bardsley TR, et al. Risk factors for placenta accreta: a large prospective cohort. Am J Pe-rinatol. 2014;31:799-804.

4. Meng X, Xie L, Song W. Comparing the diagnostic value of ultrasound and magnetic resonance imaging for placenta accreta: a systematic review and meta-analysis. Ultrasound Med Biol. 2013;39:1958-1965.

5. Sentilhes L, Ambroselli C, Kayem G, et al. Maternal outcome after conservative treatment of placenta accreta. Obstet Gynecol 2010;115(3):526-534.

6. Fitzpatrick KE, Sellers S, Spark P, et al. The management and outcomes of placenta accreta, increta, and percreta in the UK: a population-based descriptive study. BJOG. 2014;121:62-70. 
7. D’Antonio F, Iacovella C, Bhide A. Prenatal identifcation of invasive placentation using ultrasound: systematic review and meta-analysis. Ultrasound Obstet Gynecol. 2013;42(5):509-517.

8. Jauniaux E, Bhide A, Kennedy A, et al. FIGO Placenta Accreta Diagnosis and Management Expert Consensus Panel. FIGO consensus guidelines on placenta accreta spectrum disorders: Prenatal diagnosis and screening. Int J Gynaecol Obstet. 2018;140(3):274-280.

9. Sentilhes L, Kayem G, Chandraharan E, et al. FIGO Placenta Accreta Diagnosis and Management Expert Consensus Panel. FIGO consensus guidelines on placenta accreta spectrum disorders: Conservative management. Int J Gynaecol Obstet. 2018;140(3):291-298.
10. Allen L, Jauniaux E, Hobson S, et al. FIGO Placenta Accreta Diagnosis and Management Expert Consensus Panel. FIGO consensus guidelines on placenta accreta spectrum disorders: Nonconservative surgical management. Int J Gynaecol Obstet. 2018;140(3):281-290.

11. Sentilhes L, Goffnet F, Kayem G. Management of placenta accreta. Acta Obstet Gynecol Scand. 2013;92:1125-1134.

12. Silver RM, Fox KA, Barton JR, et al. Center of excellence for placenta accreta. Am J Obstet Gynecol. 2015; 212(5):560-568. 